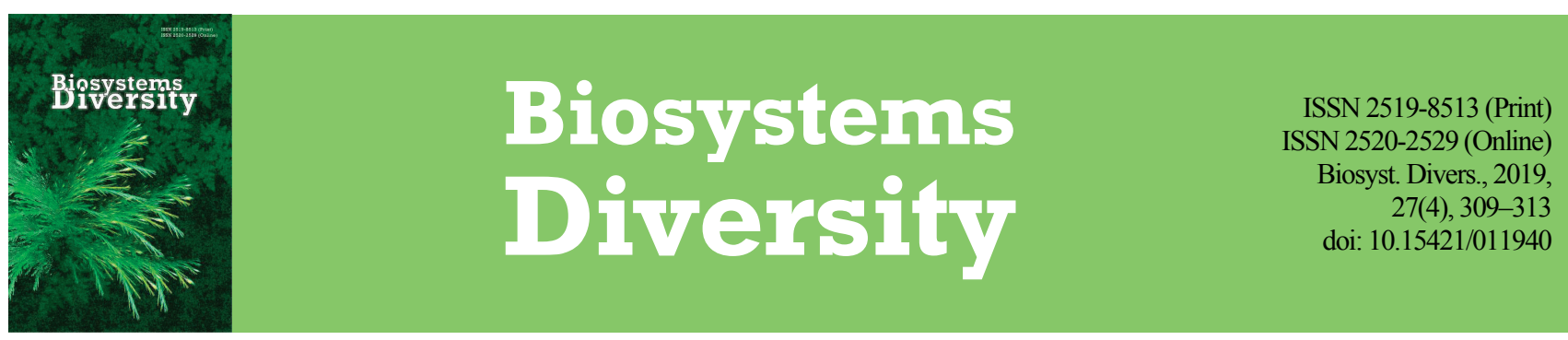

\title{
Method of individual forecasting of sow reproductive performance on the basis of a non-linear canonical model of a random sequence
}

\author{
I. P. Atamanyuk, V. Y. Kondratenko, A. S. Kramarenko, O. E. Novikov, V. Y. Lykhach
}

Mykolayiv National Agrarian University, Mykolayiv, Ukraine

Article info

Received 17.09.2019

Received in revised form

12.10.2019

Accepted 14.10.2019

Mykolayiv National

Agrarian University

Georgiy Gongadze st., 9,

Mykolayiv, 54020, Ukraine.

Tel.: +38-050-991-53-14.

E-mail:

kssnail1990@gmail.com
Atamanyuk, I. P., Kondratenko, V. Y., Kramarenko, A. S., Novikov, O. E., \& Lykhach, V. Y. (2019). Method of individual fore-
casting of sow reproductive performance on the basis of a non-linear canonical model of a random sequence. Biosystems Diversity, 27(4), 309-313. doi:10.15421/011940

Improvement of sow reproductive performance is a key factor determining the efficiency of the pig production cycle and profitability of pork production. This article presents the solution of an important scientific and practical problem of individual forecasting of sow reproduction. The population used for the present study is from a pig farm managed by the Limited Liability Company (LLC) 'Tavriys'kisvyni' located in Skadovsky district (Kherson region, Ukraine). The experimental materials used for this study consisted of 100 inds. of productive parent sows of the Large White breed.The litter size traits - the total number of piglets born (TNB), number of piglets born alive (NBA) and number of weaned piglets (NW) - were monitored in the first eight parities over an eleven year period (2007-2017). The method of the forecasting of sow litter size is developed based on the non-linear canonical model of the random sequence of a litter size change. The proposed method allows us to take maximum account of stochastic peculiarities of sow reproductive performance and does not impose any restrictions on the random sequence of a litter size change (linearity, stationarity, Markov property, monotony, etc.). The block diagram of the algorithm presented in this work reflects the peculiarities of calculation of the parameters of a predictive model. The expression for the calculation of an extrapolation error allows us to estimate the necessary volume of a priori and a posteriori information for achieving the required quality of solving the forecasting problem. The results of the numerical experiment confirmed the high accuracy of the proposed method of forecasting of sow reproduction. The method offered by us almost doubles the accuracy of forecasting of sow litter size compared to the use of the Wiener and Kalman methods. Thus, average forecast error decreases across the range of features TNB (1.71), NBA (1.68) and NW (1.25 piglets). Apparently, this may reflect a higher level of manifestation of the genetically determined level of individual sow fertility at the moment of piglet weaning. The higher adequacy of the developed mathematical model with regard to NW can be also due to the fact that the relations between sow litter size in different farrowings primarily have a non-linear character, which is taken into maximum account in our offered model. Given non-linearity, on the other hand, turns out to be a significant factor determining a lower estimation of the repeatability value for NW compared to the estimations for TNB and NBA. The use of the developed method will help to improve the efficiency of pig farming.

Keywords: pig; individual forecasting of sow reproduction; non-linear canonical model of the random sequence.

\section{Introduction}

Since the early 1990s, the improvement of the sow fertility for maximizing the number of live-born and weaned piglets per sow and year has become the main aim of pig breeding (Biermann et al., 2014). Thus, improvement of sow reproductive performance is a key factor determining the efficiency of pig production cycle and profitability of pork production. Fertility of sows is a complex feature that is determined by a whole set of factors of genetic and paragenetic nature. As for the latter, the age of a sow (i.e., number of farrowings), which determines the physiological status of an animal (growth, development of the reproductive system, body condition, etc.), perhaps most determines its reproductive functions (Schwarz \&Kopyra, 2006; Canario et al., 2006).

As a rule, sow litter size tends to rise to the 3 th-5th parities and then smoothly decline (Tantasuparuk et al., 2000; Tummaruk et al., 2000; Lavery et al., 2019) which is related to the increase of the number of stillborn piglets in later parities. The given pattern is mostly explained by excessive fatness of old sows or aging of the uterus, which, having reduced muscular tone, becomes less efficient for the farrowing process (Leenhouwers et al., 1999; Borges et al., 2005). On the other hand, the number of stillborn piglets increases with the rise of litter size, which happens primarily due to asphyxia during parturition (Zaleski \& Hacker, 1993; Canario et al., 2006). However, the individual sow fertility rate directly influences its further use in pig farming because low litter size for a number of previous farrowings increases the probability of the animal's culling and replacement with a gilt for optimization of the herd age structure to provide the maximum total number of piglets per sow and year. As stated, annual sow culling rates, on average, vary from $35 \%$ to $59 \%$ (Małopolska et al., 2018). This means that, the reproductive issues including small litter size are the reason for the culling of every third sow (Lucia et al., 2000).

Many authors have noted that between the litter size for different farrowings, there is a weak but significant phenotypic correlation which is most expressed between the pairs of consecutive parities (Roehe \& Kennedy, 1995; Radojković et al., 2018; Ye et al., 2018). Therefore, the attempt to forecast future sow litter size based on the animal's performance for the previous one or two farrowings and thus, making the decision about the appropriateness of the sow's culling and replacement, can be made with a certain level of accuracy. In Iida et al. (2015), it was stated that high fertility rates for the first and second sow farrowings can be used for the forecasting of a higher level of their lifetime performance. Methods of mathematical modeling are actively used in pig breeding for the development of optimization models:replacement model (Huirne et al., 1988), delivering the pigs to the slaughterhouse (Jørgensen, 1993), treatment decisions (e.g. regarding vaccination for disease problems) (Toft et al., 2005), feeding decisions of growing pigs (Glen, 1983; Pourmoayed et al., 2016), litter size model (Toft \& Jørgensen, 2002), etc. However, the litter size model which was offered in Toft 
\& Jørgensen (2002) and further developed in Bono et al. (2012) reflects not so much individual performance rates for each sow but estimations averaged across a herd at discrete moments of time. And they consequently aim at monitoring a general trend of change of herd fertility as a whole, based on statistical control tools such as Shewhart Control Charts and V-masks.

Thereupon, obtaining the method which allows one to forecast individual sow litter size using data about their fertility for the previous farrowings and thus, to forecast the necessity of their culling and replacement is the main aim of the work.

\section{Materials and methods}

The population used for the present study is from a pig farm managed by the Limited Liability Company (LLC) 'Tavriys'kisvyni' located in Skadovsky district (Kherson region, Ukraine). The experimental materials used for this study consisted of 100 inds. of productive parent sows of the Large White breed. The estimation of reproductive performance was conducted for each animal included in this study. The litter size traits - the total number of piglets born (TNB), number of piglets born alive (NBA) and number of weaned piglets (NW) - were monitored in the first eight parities in an eleven year period (20072017). Considering that a random sequence of sow litter size change is characterized with weak stochastic relations, to forecast future values of litter size, it is necessary to use methods and models which allow one to take into maximum account probabilistic properties and peculiarities of fertility rates.

The most general extrapolation form for solving the nonlinear prediction problem is the Kolmogorov-Gabor polynomial (Atamanyuk, 2005) but the determination of its parameters for the highest number of known values and the used order of nonlinear connections is a very difficult and laborious process (thus, for 11 already known values and the 4th order of nonlinearity, it is necessary to obtain and solve 1819 equations of partial derivatives of mean-square error of extrapolation). Thereupon, when forming realizable in practice algorithms of the forecasting, different simplifications and limitations on the properties of a random sequence are used. For example, a number of suboptimal nonlinear extrapolation methods with a limited order of a stochastic relation on the basis of approximation of a posteriori density of probabilities of an estimated vector by orthogonal Hermite polynomial expansion or in the form of the Edgeworth series is offered by Pugachev (1965). The solution to the non-stationary Kolmogorov equation (in particular, the case of the Stratanovich differential equation to describe the Markovian processes) is obtained provided that a drift coefficient is a linear function of condition, and the diffusion coefficient is equal to constant. An exhaustive solution to the optimal linear extrapolation problem for different classes of random sequences and different levels of informational support of the prediction problem (the Kolmogorov equation for random stationary sequences measured without errors; the Kalman method (Box et al., 2015) used for Markovian noisy random sequences; the Wiener-Hopf filter-extrapolator (Wiener, 1970) for noisy stationary sequences etc.) exists. But the use of simplifying assumptions considerably limits the extrapolation accuracy.

The most universal method from the point of view of limitations on the investigated random sequence

$$
\{x\}=x(i), i=\overline{1, l}
$$

$X(i)$ - number of piglets in the $i$-th farrowing, $I$ - maximum number of farrowings) is the non-linear polynomial algorithm (Atamanyuk, 2009; Atamanyuk et al., 2015):

$$
m_{x}^{(\mu, l)}(h, i)=\left\{\begin{array}{c}
M\left[X^{h}(i)\right] \text { at } \mu=0 ; \\
m_{x}^{(\mu, l-1)}(h, i)+\left(x^{l}(\mu)-m_{x}^{(\mu, l-1)}(l, \mu)\right) \beta_{h \mu}^{(l)}(i) \text { at } l \neq 1, \\
m_{x}^{(\mu-1, N)}(h, i)+\left(x^{l}(\mu)-m_{x}^{(\mu-1, N)}(l, \mu)\right) \beta_{h \mu}^{(1)}(i) \text { at } l=1 .
\end{array}\right.
$$

The expression $m_{x}^{(\mu, l)}(h, i)=M\left[X^{h}(i) / x^{v}(j), j=\overline{1, \mu-1}, v=\overline{1, N}\right.$; $\left.x^{v}(\mu), v=\overline{1, l}\right]$ for $h=1, l=N, \mu=k \quad$ is an optimal estimation $m_{x}^{(k, N)}(1, i)$ of the future value $x(i), i=\overline{k+1, I}$ provided that the values $x^{v}(j), v=\overline{1, N}, j=\overline{1, k}$ are used for the calculation of the given estima- tion. Canonical random expansion of a random sequence underlies the forecasting model:

$$
X(i)=M[X(i)]+\sum_{v=1}^{i} \sum_{\lambda=1}^{N} W_{v}^{(\lambda)} \beta_{1 v}^{(\lambda)}(i), i=\overline{1, I}
$$

Elements $W_{v}^{(\lambda)}, \beta_{h v}^{(\lambda)}(i)$ of the model are determined by recurrent relations (algorithm of parameter calculation is presented in Fig. 1):

$$
\begin{gathered}
W_{v}^{(\lambda)}=X^{\lambda}(v)-M\left[X^{\lambda}(v)\right]-\sum_{\mu=1}^{v-1} \sum_{j=1}^{N} W_{\mu}^{(j)} \beta_{\lambda \mu}^{(j)}(v)- \\
-\sum_{j=1}^{\lambda-1} W_{v}^{(j)} \beta_{\lambda v}^{(j)}(v), v=\overline{1, I} ; \\
\beta_{h v}^{(\lambda)}(i)=\frac{M\left[W_{v}^{(\lambda)}\left(X^{h}(i)-M\left[X^{h}(i)\right]\right)\right]}{M\left[\left\{W_{v}^{(\lambda)}\right\}^{2}\right]}= \\
-\frac{1}{D_{\lambda}(v)}\left\{M\left[X^{\lambda}(v) X^{h}(i)\right]-\right. \\
-M\left[X^{\lambda}(v)\right] M\left[X^{h}(i)\right]-\sum_{\mu=1}^{v-1} \sum_{j=1}^{N} D_{j}(\mu) \beta_{\lambda \mu}^{(j)}(v) \beta_{h \mu}^{(j)}(i)- \\
\left.-\sum_{j=1}^{\lambda-1} D_{j}(v) \beta_{\lambda v}^{(j)}(v) \beta_{h v}^{(j)}(i)\right\}, \lambda=\overline{1, h}, v=\overline{1, i}, h=\overline{1, N}, i=\overline{1, I} . \\
D_{\lambda}(v)=M\left[\left\{W_{v}^{(\lambda)}\right\}^{2}\right]=M\left[X^{2 \lambda}(v)\right]-M^{2}\left[X^{\lambda}(v)\right]- \\
-\sum_{\mu=1}^{v-1} \sum_{j=1}^{N} D_{j}(\mu)\left\{\beta_{\lambda \mu}^{(j)}(v)\right\}^{2}-\sum_{j=1}^{\lambda-1} D_{j}(v)\left\{\beta_{\lambda \nu}^{(j)}(v)\right\}^{2}, \lambda=\overline{1, N}, v=\overline{1, I} .
\end{gathered}
$$

where $D_{\lambda}(v)$ is a random variance coefficient of $W_{v}^{(\lambda)}$.

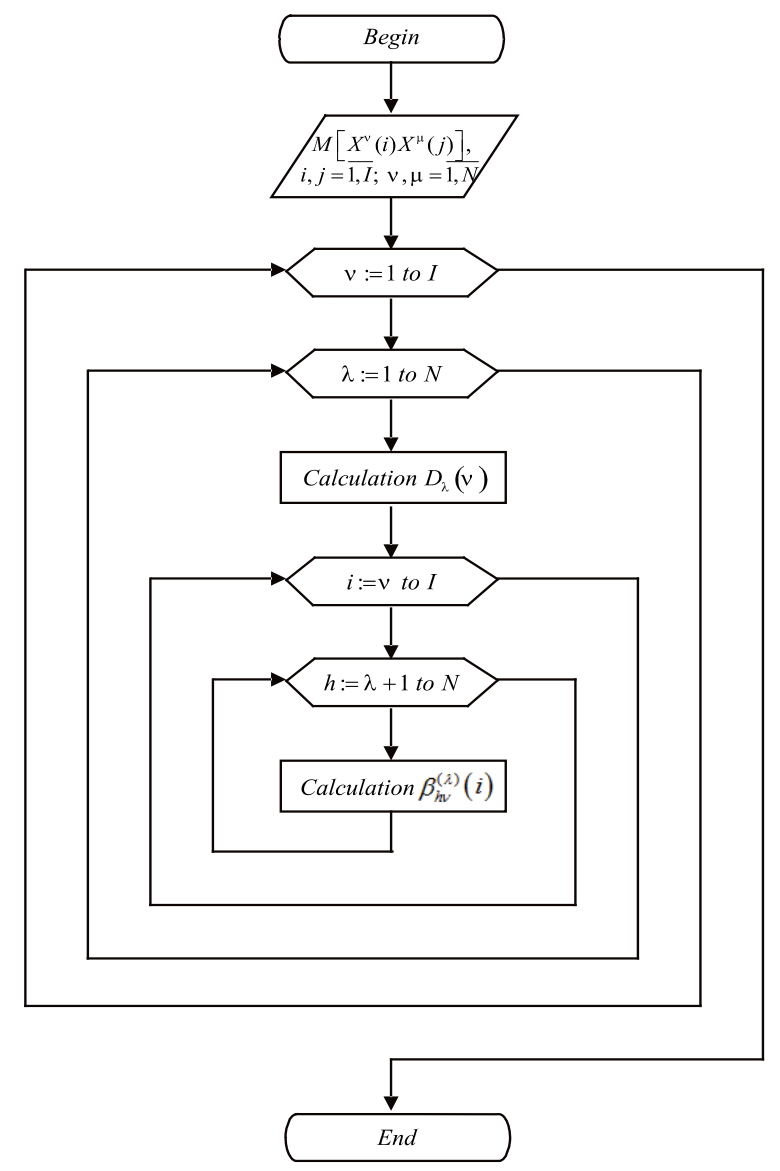

Fig. 1. A block diagram to represent the calculations of the coordinate functions and the variances of random coefficients of canonical expansion (2)

The coordinate functions $\beta_{h v}^{(\lambda)}(i), v=\overline{1, i} ; \lambda, h=\overline{1, N} ; i=\overline{1, I}$ are characterized by the properties:

$$
\beta_{h v}^{(\lambda)}(i)=\left\{\begin{array}{l}
1, \text { if }(h=\lambda) \wedge(v=i) ; \\
0, \text { if }(i<v) \vee((h<\lambda) \wedge(v=i)) .
\end{array}\right.
$$


The model (2) of the sequence $\{X\}=X(i), i=\overline{1, I}$ contains $\mathrm{N}$ arrays $\left\{W^{(\lambda)}\right\}, \lambda=\overline{1, N}$ of uncorrelated centered random coefficients $W_{i}^{(\lambda)}, \lambda=\overline{1, N}, i=\overline{1, I}$. Each of the given coefficients contains the information about the corresponding value $X^{\lambda}(i), \lambda=\overline{1, N}, i=\overline{1, I}$, and the coordinate functions $\beta_{h v}^{\lambda}(i), \lambda, h=\overline{1, N}, v, i=\overline{1, I}$ describe probabilistic relations of the order $\lambda+h$ between two different sections $v$ and $i,(v, i=\overline{1, I})$.

A canonical expansion (2) provides the mean-square error minimum of a random sequence presentation and allows the realization of a modeling sequence on a random number of intervals (depending only on the possibilities of a computer).

An expression for the sought value $m_{x}^{(k, N)}(1, i)$ can be written down in the following explicit form (Atamanyuk et al., 2012):

$$
\begin{aligned}
& m_{x}^{(k, N)}(1, i)=M[X(i)]+\sum_{j=1}^{k} \sum_{v=1}^{N}\left(x^{v}(j)-M\left[X^{v}(j)\right]\right) \times \\
& \times S_{((j-1) N+v)}^{(k N)}((i-1) N+1),
\end{aligned}
$$

where

$$
\begin{aligned}
& S_{\lambda}^{(\alpha)}(\xi)=\left\{\begin{array}{l}
S_{\lambda}^{(\alpha-1)}(\xi)-S_{\lambda}^{(\alpha-1)}(\alpha) \gamma_{k}(i), \text { at } \lambda \leq \alpha-1 ; \\
\gamma_{\alpha}(\xi), \text { for } \lambda=\alpha ;
\end{array}\right. \\
& \gamma_{\alpha}(\xi)=\left\{\begin{array}{l}
\beta_{1,[\alpha / N]+1}^{\left(\bmod _{N}(\alpha)\right)}([\alpha / N]+1), \text { for } \xi \leq k N ; \\
\beta_{1,[\alpha / N]+1}^{\left(\bmod _{N}(\alpha)\right)}(i), \text { if } \xi=(i-1) N+1 .
\end{array}\right.
\end{aligned}
$$

In expression (8) [.] is the operation of rounding.

A canonical expansion of a posteriori random sequence $\{\mathrm{X}\}$, provided that the first $\mathrm{k}$ of values are fixed, will be written as:

$$
\begin{aligned}
X^{(k, N)}(i)= & X\left(i / x^{\nu}(j), v=\overline{1, N}, j=\overline{1, k}\right)=m_{x}^{(k, N)}(1, i)+ \\
& +\sum_{\mu=k+1}^{i} \sum_{\lambda=1}^{N} W_{\mu}^{(\lambda)} \beta_{1 \mu}^{(\lambda)}(i), i=\overline{k+1, I} .
\end{aligned}
$$

The error of a single extrapolation at known values $x^{v}(j), v=\overline{1, N}, j=\overline{1, k}$ is determined by the formula:

$$
\begin{aligned}
& \delta\left[i / x^{v}(j), v=\overline{1, N}, j=\overline{1, k}\right]=m_{x}^{(k, N)}(1, i)-x^{(k, N)}(i), \\
& i=\overline{k+1, I} .
\end{aligned}
$$

where $x^{(k, N)}(i), i=\overline{k+1, I}$ are the future values of extrapolated realization.

The realization $x^{(k, N)}(i), \mathrm{i}=\overline{k+1, I}$ in the area of prediction develops randomly, that is why it is impossible to specify its exact values. However, the model (9) is an accurate description of a stochastic development of the realization $x^{(k, N)}(i), \mathrm{i}=\overline{k+1, I}$ that allows one to reduce the expression (10) to the form:

$$
\begin{aligned}
& \Delta\left[i / x^{v}(j), v=\overline{1, N}, j=\overline{1, k}\right]=m_{x}^{(k, N)}(1, i)- \\
& -X^{(k, N)}(i), i=\overline{k+1, I} .
\end{aligned}
$$

Thus, a single extrapolation error has stochastic character determined by a random nature of the studied sequence.

The application to (11) of the operation of mathematical expectation taking into account the expression (9) allows one to determine the systematic constituent of the error of a single extrapolation:

$$
\begin{gathered}
S\left[i / x^{v}(j), v=\overline{1, N}, j=\overline{1, k}\right]=M\left[\Delta\left(i / x^{v}(j), j=\overline{1, k}\right)\right]= \\
=\sum_{\mu=k+1}^{i} \sum_{\lambda=1}^{N} M\left[W_{\mu}^{(\lambda)}\right] \beta_{1 \mu}^{(\lambda)}(i)=0, i=\overline{k+1, I} .
\end{gathered}
$$

The last result shows that the predictive models (1) and (6) which provide the receiving of unbiased estimates of future values for each predictable realization. This also implies that the algorithm provides unbiasedness on an average:

$$
\begin{aligned}
& S^{(k, N)}(i)=M\left[S\left(i / X^{v}(j), v=\overline{1, N}, j=\overline{1, k}\right)\right]=0, \\
& i=\overline{k+1, I} .
\end{aligned}
$$

The estimation of the significance of a random constituent of the single extrapolation error is its variance:

$$
\begin{aligned}
& D_{\Delta}\left[i / x^{v}(j), v=\overline{1, N}, j=\overline{1, k}\right]= \\
& =M\left[\left(\Delta\left[i / x^{v}(j), v=\overline{1, N}, j=\overline{1, k}\right]-\right.\right.
\end{aligned}
$$

$$
\begin{aligned}
& \left.\left.-S\left[i / x^{v}(j), v=\overline{1, N}, j=\overline{1, k}\right]\right)^{2}\right]= \\
& =M\left[\left(\sum_{\mu=k+1}^{i} \sum_{\lambda=1}^{N} W_{\mu}^{(\lambda)} \beta_{1 \mu}^{(\lambda)}(i)\right)^{2}\right]= \\
& =\sum_{\mu=k+1}^{i} \sum_{\lambda=1}^{N} D_{\lambda}(\mu)\left\{\beta_{1 \mu}^{(\lambda)}(i)\right\}^{2}, i=\overline{k+1, I} .
\end{aligned}
$$

As $D_{\Delta}\left[i / x^{v}(j), v=\overline{1, N}, j=\overline{1, k}\right]$ doesn't depend on the specific values of extrapolated realization, its averaging by the multitude of extrapolations allows one to write down the expression for variance of a posteriori random sequence of

$$
\begin{aligned}
& D_{\Delta}^{(k, N)}(i)=M\left[D_{\Delta}\left[i / X^{v}(j), v=\overline{1, N}, j=\overline{1, k}\right]\right]= \\
& =\sum_{\mu=k+1}^{i} \sum_{\lambda=1}^{N} D_{\lambda}(\mu) \beta_{1 \mu}^{(\lambda)}(i) .
\end{aligned}
$$

Taking (13) and (15) into account, the expression for the meansquare error of prediction will be written down as:

$$
\begin{gathered}
E_{x}^{(k, N)}(i)=\left[S^{(k, N)}(i)\right]^{2}+D_{\Delta}^{(k, N)}(i)= \\
=D_{\Delta}^{(k, N)}(i)=\sum_{\mu=k+1}^{i} \sum_{\lambda=1}^{N} D_{\lambda}(\mu) \beta_{1 \mu}^{(\lambda)}(i), i=\overline{k+1, I} .
\end{gathered}
$$

Thus, the mean-square error of the prediction with the help of algorithm (1) and (6) is equal to the variance of a posteriori random sequence.

Forecasting method. The application of models and algorithms, used to predict litter size, assumes the realization of the following stages:

Stage 1. Statistical data collection about a studied random sequence of litter size change.

Stage 2. The estimation of moment functions $M\left[X^{\lambda}(i)\right]$, $M\left[X^{\lambda}(i) X^{h}(j)\right]$ on the basis of accumulated realizations of a random sequence of litter size change.

Stage 3. The forming of canonical expansion (2) based on the block diagram (Fig. 1) for the studied sequence.

Stage 4 . The prediction of the future values of litter size based on the models (1) and (6).

Stage 5. The estimation of the quality to solve the problem of predicting litter size with the help of the expression (16).

The results of a numerical experiment. To carry out comparative analysis based on the statistical data a numerical experiment was carried out with the use of the linear Wiener method, the fifth-order Kalman method (stochastic relations above the 5th order are not significant for the investigated statistical data) and with the help of an algorithm based on the predictive model (1) and (6) of the fifth-order nonlinear relation. To determine the litter size in the 8th farrowing, information on the seven previous farrowings was used.

The forecasting model (6) for set conditions of the experiment takes the following form:

$$
m_{x}^{(7,5)}(1,8)=M[X(8)]+\sum_{j=1}^{7} \sum_{v=1}^{5}\left(x^{v}(j)-M\left[X^{v}(j)\right]\right) S_{(j)}^{(v)}(8),
$$

In Tables 1,2 and 3 the values of weighting coefficients $S_{(j)}^{(v)}(8), v=\overline{1,5}, j=\overline{1,7}$ for the case of TNB, NBA and NW forecasting are presented. To determine each multitude of weighting coefficients $S_{(j)}^{(v)}(8), v=\overline{1,5}, j=\overline{1,7}, 594$ coordinate functions of the mathematical model (2) of the random sequence of sow litter size change were used.

\section{Table 1}

Values of weighting coefficients $S_{(j)}^{(v)}(8), v=\overline{1,5}, j=\overline{1,7}$ for the case of TNB forecasting

\begin{tabular}{cccccccc}
\hline$v$ & \multicolumn{7}{c}{$j$} \\
\cline { 2 - 8 } & 1 & 2 & 3 & 4 & 5 & 6 & 7 \\
\hline 1 & -11.49 & -2.48 & 31.72 & 45.85 & -26.37 & 2.63 & 20.13 \\
2 & 2.79 & -0.26 & -4.61 & -10.53 & 6.67 & -0.37 & -4.61 \\
3 & -0.298 & 0.096 & 0.309 & 1.113 & -0.758 & 0.027 & 0.482 \\
4 & 0.01478 & -0.00791 & -0.00938 & -0.05529 & 0.03996 & -0.00111 & -0.02344 \\
5 & -0.00028 & 0.00021 & 0.00009 & 0.00104 & -0.00079 & 0.00002 & 0.00042 \\
\hline
\end{tabular}

The results of the experiment (Table 4) show the high accuracy of prediction based on the developed technology in comparison with the Wiener method with the use of nonlinear stochastic relations and in 
comparison with the Kalman method on account of an essential increase of a posteriori information which is used for prediction.

\section{Table 2}

Values of weighting coefficients $S_{(j)}^{(v)}(8), v=\overline{1,5}, j=\overline{1,7}$

for the case of NBA forecasting

\begin{tabular}{cccccccc}
\hline$v$ & \multicolumn{7}{c}{$j$} \\
\cline { 2 - 8 } & 1 & 2 & 3 & 4 & 5 & 6 & 7 \\
\hline 1 & -6.05 & 46.47 & 8.95 & 7.67 & -31.67 & 5.77 & -3.68 \\
2 & 1.61 & -13.16 & -3.64 & -2.07 & 8.24 & -1.55 & 0.74 \\
3 & -0.191 & 1.738 & 0.561 & 0.251 & -0.993 & 0.185 & -0.061 \\
4 & 0.01121 & -0.10836 & -0.03724 & -0.01419 & 0.05615 & -0.0103 & 0.0021 \\
5 & -0.00027 & 0.00257 & 0.00089 & 0.00031 & -0.00121 & 0.0002 & -0.0000 .
\end{tabular}

\section{Table 3}

Values of weighting coefficients $S_{(j)}^{(v)}(8), v=\overline{1,5}, j=\overline{1,7}$

for the case of NW forecasting

\begin{tabular}{cccccccc}
\hline$v$ & \multicolumn{7}{c}{$j$} \\
\cline { 2 - 8 } & 1 & 2 & 3 & 4 & 5 & 6 & 7 \\
\hline 1 & 0.43 & 11.52 & 127.14 & 11.73 & -92.82 & 6.19 & -46.65 \\
2 & -1.12 & -4.16 & -25.83 & -3.71 & 29.97 & -3.18 & 9.16 \\
3 & 0.325 & 0.613 & 2.425 & 0.531 & -4.517 & 0.621 & -0.718 \\
4 & -0.03354 & -0.03949 & -0.10124 & -0.03545 & 0.32094 & -0.05281 & 0.014608 \\
5 & 0.00115 & 0.00091 & 0.00134 & 0.00091 & -0.00868 & 0.00164 & 0.000399 \\
\hline
\end{tabular}

\section{Table 4}

Average forecast error estimates

for different sow litter size traits, piglets

\begin{tabular}{lccc}
\hline \multicolumn{1}{c}{ Method } & TNB & NBA & NW \\
\hline Wiener & 3.10 & 2.80 & 2.50 \\
Kalman & 2.90 & 2.70 & 2.45 \\
Developed method & 1.71 & 1.68 & 1.25 \\
\hline
\end{tabular}

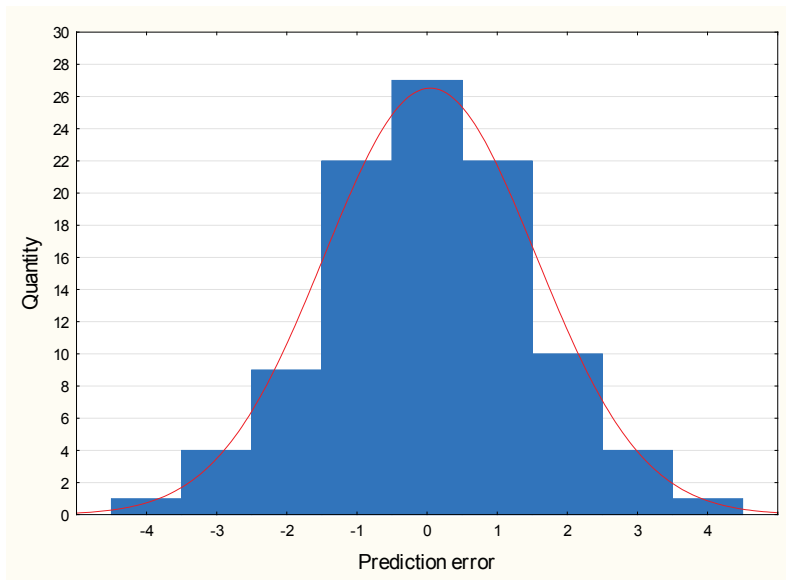

Fig. 2. Histogram of the forecast error rate for the case of NW prediction

The estimation of the density of extrapolation error distribution (Fig. 2) for the case of NW forecasting has a distribution law close to the normal one, which indicates the maximum accuracy of solving of the forecasting problem using the proposed method.

\section{Discussion}

A method of solving an important task of forecasting of sow litter size is offered. The forecasting method, as well as an underlying canonical model, doesn't impose any limitations on the properties of a random sequence of change of sow reproductive performance (linearity, stationarity, linearity, Markov property, monotony, etc.). Taking into full account the stochastic peculiarities of fertility rates allows one to achieve maximum quality of solving of a forecasting problem. The results of a numerical experiment confirmed the high accuracy characteristics of a predictive model.

The method presented by us almost doubles the accuracy of forecasting of sow litter size compared to the use of the Wiener and Kalman methods. Thus, average forecast error decreases across the range of features TNB (1.71), NBA (1.68) and NW (1.25 piglets). Apparently, this may reflect a higher level of manifestation of the genetically determined level of individual sow fertility at the moment of piglet weaning. Earlier, a lower level of entropy concerning the number of weaned piglets was discovered by us when analyzing the variability of litter size of the sows of large white and Ukrainian meat breeds, especially for the 4-7th farrowings (Kramarenko \& Lugovoy, 2013). Thus, it is appropriate to use the number of weaned piglets as the main indicator when estimating sow reproductive performance.

The higher adequacy of the developed mathematical model with regard to NW can be also due to the factor that the relations between sow litter size in different farrowings primarily have a non-linear character, which is taken into maximum account in our offered model. The given non-linearity, on the other hand, turns out to be a significant factor determining a lower estimation of the repeatability value for NW compared to the estimations for TNB and NBA (Strang \& Smith, 1979; Szyndler-Nędza, 2016).

\section{Conclusion}

A method of the forecasting of sow litter size is developed based on the non-linear canonical model of the random sequence of a litter size change. The proposed method allows us to take maximum account of stochastic peculiarities of sow reproductive performance and doesn't impose any restrictions on the random sequence of a litter size change (linearity, stationarity, Markov property, monotony, etc.). The expression for the calculation of an extrapolation error allows us to estimate the necessary volume of a priori and a posteriori information for achieving the required quality of solving a forecasting problem. The results of the numerical experiment confirmed the high accuracy of the proposed method of forecasting of sow reproduction. The use of the developed method will help to improve the efficiency of pig farming.

Financial support was received from the Ministry of Education and Science of Ukraine (state registration number 0119U001042).

\section{References}

Atamanyuk, I. P. (2005). Algorithm of extrapolation of a nonlinear random process on the basis of its canonical decomposition. Cybernetics and Systems Analysis, 41(2), 267-273.

Atamanyuk, I. P. (2009). Optimal polynomial extrapolation of realization of a random process with a filtration of measurement errors. Journal of Automation and Information Sciences, 41(8), 38-48.

Atamanyuk, I. P., Kondratenko, V. Y., Kozlov, O. V., \& Kondratenko, Y. P. (2012). The algorithm of optimal polynomial extrapolation of random processes. In: International conference on modeling and simulation in engineering, economics and management. Springer, Berlin, Heidelberg. Pp. 78-87.

Atamanyuk, I., Kondratenko, Y., Shebanin, V., \& Mirgorod, V. (2015). Method of polynomial predictive control of fail-safe operation of technical systems. In: The experience of designing and application of CAD systems in microelectronics. IEEE, Lviv, Polyana. Pp. 248-251.

Biermann, A. D. M., Pimentel, E. C. G., Tietze, M., Pinent, T., \& König, S. (2014). Implementation of genetic evaluation and mating designs for the endangered local pig breed 'Bunte Bentheimer'. Journal of Animal Breeding and Genetics, 131(1), 36-45.

Bono, C., Cornou, C., \& Kristensen, A. (2012). Dynamic production monitoring in pig herds I: Modeling and monitoring litter size at herd and sow level. Livestock Science, 149(3), 289-300.

Borges, V. F., Bernardi, M. L., Bortolozzo, F. P., \& Wentz, I. (2005). Risk factors for stillbirth and foetal mummification in four Brazilian swine herds. Preventive Veterinary Medicine, 70(3-4), 165-176.

Box, G. E., Jenkins, G. M., Reinsel, G. C., \& Ljung, G. M. (2015). Time series analysis: Forecasting and control. John Wiley \& Sons Inc., Hoboken, New Jersey.

Canario, L., Cantoni, E., Le Bihan, E., Caritez, J. C., Billon, Y., Bidanel, J. P., \& Foulley, J. L. (2006). Between-breed variability of stillbirth and its relationship with sow and piglet characteristics. Journal of Animal Science, 84(12), 3185-3196.

Glen, J. (1983). A dynamic programming model for pig production. Journal of the Operational Research Society, 34, 511-519. 
Huirne, R. B. M., Hendriks, T. H., Dijkhuizen, A. A., \& Giesen, G. W. J. (1988). The economic optimisation of sow replacement decisions by stochastic dynamic programming. Journal of Agricultural Economics, 39(3), 426-438.

Iida, R., Piñeiro, C., \& Koketsu, Y. (2015). High lifetime and reproductive performance of sows on southern European Union commercial farms can be predicted by high numbers of pigs born alive in parity one. Journal of Animal Science, 93(5), 2501-2508.

Jørgensen, E. (1993). The influence of weighing precision on delivery decisions in slaughter pig production. Acta Agriculturae Scandinavica, Section A: Animal Science, 43(3), 181-189.

Kramarenko, S. S., \& Lugovoy, S. I. (2013). Ispol'zovanie entropiyno-informatsionnogo analiza dlya otsenki vosproizvoditel'nykh kachestv svinomatok [The use of entropy and informational analysis for the estimation of sow reproductive performance]. The Altai State Agrarian University Herald, 107, 58-62 (in Russian).

Lavery, A., Lawlor, P. G., Magowan, E., Miller, H. M., O’Driscoll, K., \& Berry, D. P. (2019). An association analysis of sow parity, live-weight and back-fat depth as indicators of sow productivity. Animal, 13(3), 622-630.

Leenhouwers, J. I., van der Lende, T., \& Knol, E. F. (1999). Analysis of stillbirth in different lines of pig. Livestock Production Science, 57(3), 243-253.

Lucia Jr., T., Dial, G. D., \& Marsh, W. E. (2000). Lifetime reproductive performance in female pigs having distinct reasons for removal. Livestock Production Science, 63(3), 213-222.

Małopolska, M. M., Tuz, R., Lambert, B. D., Nowicki, J., \& Schwarz, T. (2018). The replacement gilt: Current strategies for improvement of the breeding herd. Journal of Swine Health and Production, 26(4), 208-214.

Pourmoayed, R., Nielsen, L. R., \& Kristensen, A. R. (2016). A hierarchical Markov decision process modelling feeding and marketing decisions of growing pigs. European Journal of Operational Research, 250(3), 925-938.

Pugachev, V. S. (1965). Theory of random functions. Pergamon, New York.

Radojković, D., Savić, R., Popovac, M., Radović, Č., \& Gogić, M. (2018). The share of variance components and correlations between sow production traits in different treatments of the litter size (The repeatability and multi-trait models). Contemporary Agriculture, 67(3-4), 207-214.
Roehe, R., \& Kennedy, B. W. (1995). Estimation of genetic parameters for litter size in Canadian Yorkshire and Landrace swine with each parity of farrowing treated as a different trait. Journal of Animal Science, 73(10), 2959-2970.

Schwarz, T., Kopyra, M. (2006). Influence of age on insemination process, and reproductive performance in sows. Animal Science Papers and Reports, 24(suppl. 3), 229-239.

Strang, G. S., \& Smith, C. (1979). A note on the heritability of litter traits in pigs. Animal Production, 28(3), 403-406.

Szyndler-Nędza, M. (2016). Coefficients of repeatability for colostrum and milk composition of PLW and PL sows over three consecutive lactations. Livestock Science, $185,56-60$

Tantasuparuk, W., Lundeheim, N., Dalin, A. M., Kunavongkrit, A., \& Einarsson, S. (2000). Reproductive performance of purebred Landrace and Yorkshire sows in Thailand with special reference to seasonal influence and parity number. Theriogenology, 54(3), 481-496.

Toft, N., \& Jørgensen, E. (2002). Estimation of farm specific parameters in a longitudinal model for litter size with variance components and random dropout. Livestock Production Science, 77(2-3), 175-185.

Toft, N., Kristensen, A., \& Jørgensen, E. (2005). A framework for decision support related to infectious diseases in slaughter pig fattening units. Agricultural Systems, 85(2), 120-137.

Tummaruk, P., Lundeheim, N., Einarsson, S., \& Dalin, A. M. (2000). Reproductive performance of purebred Swedish Landrace and Swedish Yorkshire sows: I. Seasonal variation and parity influence. Acta Agriculturae Scandinavica Section A - Animal Science, 50(3), 205-216.

Wiener, N. (1970). Extrapolation, interpolation, and smoothing of stationary time series. MIT Press, New-York.

Ye, J., Tan, C., Hu, X., Wang, A., \& Wu, Z. (2018). Genetic parameters for reproductive traits at different parities in Large White pigs. Journal of Animal Science, 96(4), 1215-1220.

Zaleski, H. M., \& Hacker, R. R. (1993). Effect of oxygen and neostigmine on stillbirth and pig viability. Journal of Animal Science, 71(2), 298-305. 